\title{
2 Effects of wildfires on environmental variability: a comparative analysis using different spectral indices, patch metrics and thematic resolutions
}

\author{
5 F. Javier Lozano • Susana Suárez-Seoane • \\ 6 Estanislao De Luis
}

Received: 17 January 2009/ Accepted: 11 January 2010

(C) Springer Science+Business Media B.V. 2010
Abstract Knowledge of environmental variability and how it is affected by disturbance is crucial for understanding patterns of biodiversity and determining adequate conservation strategies. The aim of this study is to assess environmental variability in patches undergoing post-fire vegetation recovery, identifying trends of change and their relevant drivers. We particularly evaluate: the value of three spectral indices derived from Landsat satellite data [Normalized Burn Ratio (NBR), Normalized Difference Vegetation Index (NDVI) and Wetness Component of the Tasseled Cap Transformation (TCW)] for describing secondary succession; the effectiveness of three metrics (diversity, evenness and richness) as indicators of patch variability; and how thematic resolution can affect the perception of environmental variability patterns. While the system was previously characterised as highly resilient from estimations of vegetation cover, here we noted that more time is required to fully recover pre-fire environmental variability. Using mean diversity as indicator of patch variability, we found similar patterns of temporal change for the three spectral indices (NBR, NDVI and TCW). Analogous conclusions could be drawn for richness and evenness.

F. J. Lozano · S. Suárez-Seoane $(\bowtie) \cdot$ E. De Luis Área de Ecología, Departamento de Biodiversidad y Gestión Ambiental, Facultad de Ciencias Biológicas y Ambientales, Universidad de León, Campus de Vegazana s/n, 24071 León, Spain

e-mail: s.seoane@unileon.es
Patch variability, measured as diversity, showed consistent patterns across thematic resolutions, although values increased with the number of spectral classes. However, when the variance of diversity was plotted against thematic resolution, different scale dependencies were detected for those three spectral indices, yielding a dissimilar perception of patch variability. In general terms, NDVI was the best performing spectral index to assess patterns of vegetation recovery, while TCW was the worst. Finally, burned patches were classified into three classes with similar trends of change in environmental variability, which were strongly related to fire severity, elevation and vegetation type.

Keywords Diversity · Richness - Evenness · Landsat · Disturbance $\cdot$ Post-fire recovery

\section{Introduction}

Understanding patterns of species diversity and their causes is a traditional theme in ecology (Peet 1974; Huston 1994). Nevertheless, in the current context of loss of biodiversity and decreasing supply of ecosystem services (Schröter et al. 2005), it has additionally become an urgent matter of environmental, social and political concern. The occurrence of a species at a site depends on environmental variability, which

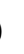

\begin{tabular}{|l|lll|}
\hline Journal : Medium 10980 & Dispatch : 16-1-2010 & Pages : 14 \\
Article No. : 9453 & $\square$ LE & $\square$ TYPESET \\
MS Code : LAND-09-1926 & $\checkmark \mathrm{CP}$ & $\searrow$ DISK \\
\hline
\end{tabular}


determines: (1) the range of suitable habitats; (2) their spatial configuration, which influences ecological processes as migration or competition; and (3) their variation over time (Dufour et al. 2006), which is associated with disturbance and succession. Disturbance has been assumed to be one of the most important factors driving species diversity ("intermediate disturbance hypothesis" by Connell 1978 and others), even if this relationship has been demonstrated as less consistent than expected (Mackey and Currie 2001) and dependent upon the spatial scale of measure (Hammer and Hill 2000; Dumbrell et al. 2008). Consequently, a better knowledge of environmental variability at varying scales, dynamics and drivers of change are crucial for understanding diversity patterns in disturbed landscapes and determining adequate conservation strategies.

In the Mediterranean Region, fire is probably the main disturbance (Lavorel 1999), shaping landscapes at different spatio-temporal scales (De Luis et al. 2008). In this area fire frequency has increased (Moreno et al. 1998; Pausas and Vallejo 1999) as a consequence of climatic factors (i.e. coincidence of hot and dry seasons and precipitation variability). Another factor is the accumulation of fuel loads subsequent to land abandonment, which results in landscape homogenization (Suárez-Seoane et al. 2002). Fire has a complex effect on vegetation regeneration, mainly due to differential responses to fire regimes (Wittenberg et al. 2007; Groeneveld et al. 2008). In this sense, Keeley et al. (2005) evaluated four hypothesis (see also Bond and van Wilgen 1996) finding that post-fire recovery patterns are determined by: (1) fire severity and post-fire fluctuations in precipitation ("eventdependent hypothesis"); (2) length of the fire free period, which affects reproductive failure and fuel accumulation ("fire interval hypothesis"); (3) internal density-dependent control, which regulates the change from herbs to woody species ("self-regulatory hypothesis"); and (4) extrinsic environmental factors that vary spatially ("environmental-filter hypothesis"). As a result of both disturbance and succession working in changing conditions, landscape becomes a heterogeneous mosaic of patches with different burning histories, which may enhance its biodiversity (Keeley et al. 2005). Since recurrence is high and recovery is quick (due to resprouting abilities or seed bank persistence), Mediterranean mosaics are highly dynamic (Trabaud and Galtié 1996, Díaz-Delgado and Pons 2001).
Furthermore, the perception of landscape complexity (i.e. patterns and associated processes) can be problematic because it depends on mapping decisions (Arnot et al. 2004). Landscape pattern measures may vary depending upon choices on spatial, temporal and thematic scales (Levin 1992; Wu et al. 2002; Wu 2004; Saura 2004). However, whilst ecologists are aware of the effect of spatio-temporal scales, few studies have investigated how thematic resolution affects the understanding of the reality. In this sense, Bailey et al. (2007a, b) and Buyantuyev and Wu (2007) demonstrated that different spatial pattern characteristics can be identified at different thematic resolutions. Since there is no single optimal thematic resolution in geospatial information, multiscale analyses based on biological traits are required when assessing relationships between landscape structure and species behaviour (Baudry and Burel 1997). For example, coarse thematic resolutions are suitable when analyzing highly mobile or generalist species, which perceive less detail in landscape. By contrast, detailed resolutions are more appropriate for species with small movement capacity or with a preference for homogeneous habitats, which perceive more landscape classes (Suárez-Seoane and Baudry 2002).

Classical studies monitor a small number of local fire events for a few years. This make difficult to infer patterns at larger spatial and temporal scales (Röder et al. 2008). At the present, the use of spectral indices derived from multi-temporal satellite data is becoming widespread to assess long temporal changes in full sets of landscape elements. In the study area, fire scar mapping (Lozano et al. 2007a), fire risk modelling (Lozano et al. 2007b, 2008) and vegetation recovery (Lozano et al. 2005) have been successfully characterized using three spectral indices: Normalized Burn Ratio (NBR) (Key and Benson 1999), Normalized Difference Vegetation Index (NDVI) (Rouse et al. 1973) and Wetness Component of the Tasseled Cap Transformation (TCW) (Crist and Cicone 1984). NBR is applied for fire mapping, fire risk modelling and severity estimation. It maximizes reflectance changes related to fire events, since nearinfrared reflectance (NIR) decreases due to vegetation removal and mid-infrared reflectance (MIR) increases with the amount of bare soil. NDVI is likely the most widely utilized index in vegetation applications, showing reasonably good results in all phases of the fire cycle. It separates green vegetation from other

$\begin{array}{lll}\text { Journal : Medium 10980 } & \text { Dispatch : } \mathbf{1 6 - 1 - 2 0 1 0} & \text { Pages : } \mathbf{1 4} \\ \text { Article No. : } \mathbf{9 4 5 3} & \square \text { LE } & \square \text { TYPESET } \\ \text { MS Code : LAND-09-1926 } & \checkmark \text { CP } & \checkmark \text { DISK }\end{array}$


surfaces because chlorophyll absorbs red light and reflects NIR wavelengths. TCW is one of the bestperforming spectral indices for monitoring post-fire recovery. It contrasts visible and NIR wavelengths (where light absorption by water is low) to SWIR (short-wave infrared) and MIR bands (where that absorption is much significant). In most cases, spectral indices are used as continuous values to characterize vegetation, or converted into Boolean variables by means of the application of particular thresholds to map different landscape elements. Here we explore the advantages of reclassifying spectral values into several classes to describe landscape under different thematic resolutions, avoiding the uncertainty problems associated with misclassification in categorical maps (Shao and Wu 2008). These data will form the basis for calculating patch variability by applying different metrics based in estimations of diversity, since they might give rise to different conclusions (Yue et al. 2005). Diversity metrics (Magurran 2004) have been widely used when characterizing post-fire recovery at a local scale (e.g. Calvo et al. 2002; Arnan et al. 2006) but they have been used less often in combination with spectral indices at a large scale.

The general aim of this study is to detect changes in environmental variability in patches affected by post-fire recovery, evaluating the effect of applying different patch metrics, Landsat spectral indices and thematic resolutions. More specifically, we try to: (1) Compare the effectiveness of each spectral index for assessing vegetation recovery and then environmental variability (measured as diversity and its components: richness and evenness); (2) Evaluate the role of thematic scale (i.e. reclassification choices to define spectral classes) on the results, looking for scale dependencies; (3) Identify types of temporal change in patch variability and their environmental drivers.

Study area

The study area is the Natural Park of Lago de Sanabria y Alrededores, in north-western Spain (Fig. 1), which comprises about 23,000 ha. Landscape pattern is patchy as a consequence of a long history of fire events and human activities. At elevations ranging from 900 to 1,300 m.a.s.l. (where most of local population lives), woodlots of Quercus pyrenaica and riparian communities of Salix spp. occur in a matrix of mixed shrubland (mainly Erica spp. and Genista spp.). At higher elevations (1,3002,100 m.a.s.1.), where topography is steep, the main landscape element is a fire-adapted heathland community dominated by Erica australis and, to a lower extent, Calluna vulgaris. Fire events, the main problem for wildlife conservation, take place very frequently during early spring (mid-late March) and summer (July to late September). Fire ignition is mainly attributed to the local population (about $90 \%$, Gutierrez, pers. com.), which has been using fire for centuries to manage vegetation. During the study period (1991-2005), $24.90 \%$ of the area was burned once, $4.75 \%$ twice, $0.40 \%$ three times and $0.05 \%$ up to four times. See Lozano et al. (2007b) for a more detailed description.

Fig. 1 Location of the study area in Spain. The figure shows heathland patches, the landscape elements most affected by fire, superimposed on shaded relief
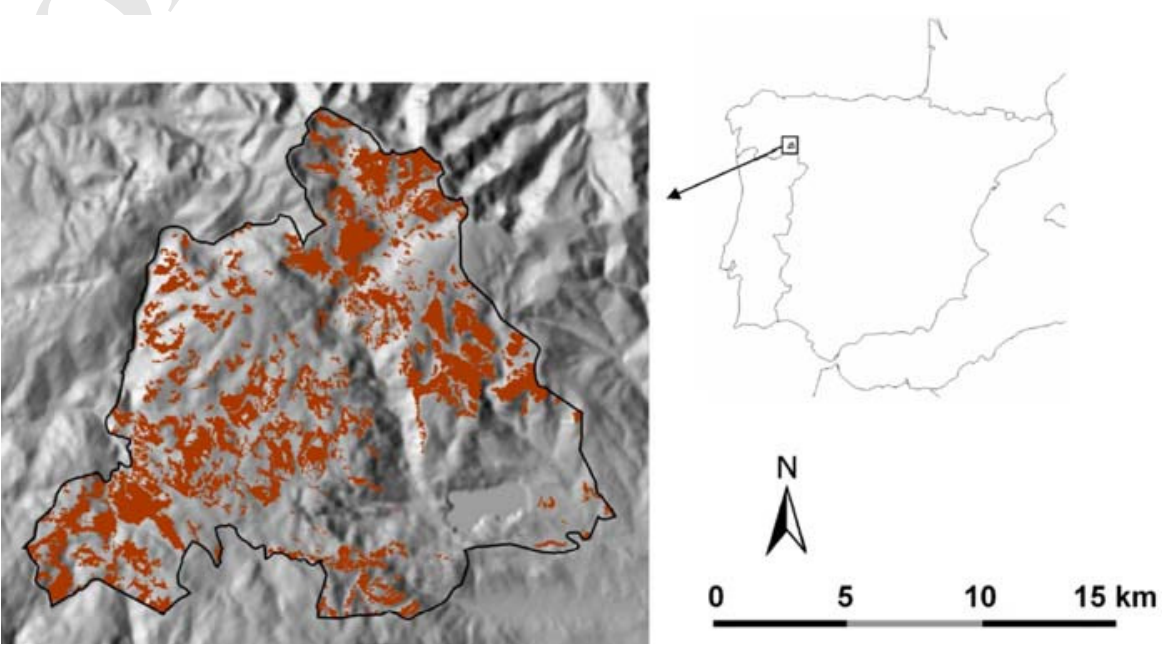

\begin{tabular}{|l|lll|}
\hline & Journal : Medium 10980 & Dispatch : 16-1-2010 & Pages : 14 \\
Article No. : 9453 & $\square$ LE & $\square$ TYPESET \\
MS Code : LAND-09-1926 & $\checkmark$ CP & $\checkmark$ DISK \\
\hline
\end{tabular}




\section{Materials and methods}

A general scheme of the methodology developed in this paper can be found in Fig. 2.

Satellite data, maps of burned areas and patch selection

One Landsat image was acquired for each year throughout the period 1991-2005 (eleven TM and four ETM + images) covering the Natural Park. Most of the images were taken in late summer (August and September) in order to consider the majority of the burning season. Geometric correction (Palá and Pons 1995) was based on ground control points (at least 50) defined on the un-referenced images $(50 \times 50 \mathrm{~km})$ with the support of orto-photographs at $70 \mathrm{~cm}$ (year 2000). $60 \%$ of the points were used to estimate the geometric fit (second order 3D polynomial) and the remaining $40 \%$ for validation purposes. The resampling option was the Nearest Neighbor Algorithm. The sub-pixel georectified images were then radiometrically corrected using the algorithms proposed by Markham and Barker (1986) and Moran et al. (1992). Atmospheric correction was based on the default transmittance method proposed by Chávez (1996). Down-welling transmittance values for bands 5 and 7 were taken from Gilabert et al. (1994), whose study area had similar atmospheric conditions to our site. Topographic correction was based on a non-

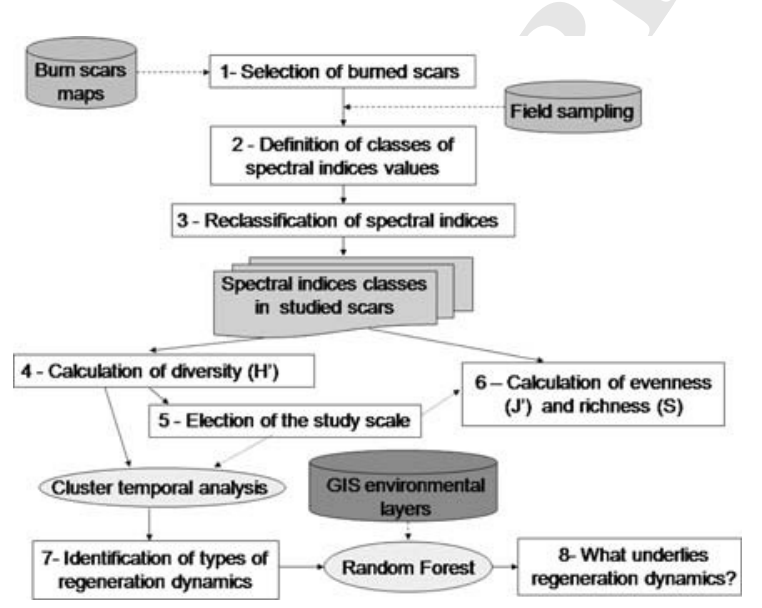

Fig. 2 General schematic representation of the methodology. Steps, databases and statistical analysis are represented in white rectangular boxes, light-gray cylinders and dark-gray ovals, respectively lambertian empirical model, the C-correction method (Riaño et al. 2003), derived from the approach of Teillet et al. (1982). Radiometric normalisation of the time-series using pseudo-invariant scene features (Hall et al. 1991) enabled a reliable intercalibration between TM and ETM + images to a common reference image.

Maps of burned areas for 1992-2005 (Lozano et al. 2007a) were derived from Landsat images by means of the differenced Normalized Burned Ratio (dNBR). Overall accuracy was $88.39 \%$, commission error $10.09 \%$ and omission error $14.37 \%$. Patches were defined as spatial aggregations of pixels with the same fire history (i.e. recurrence and year of fire event). Spatial continuity within a patch is maintained considering the eight neighbors of each pixel. A sample of ten patches was randomly selected for each year. We only considered patches burned once during the study period, since the effect of fire recurrence on environmental variability is not the subject of this paper. Due to low fire occurrence, years 1993, 1996, 2003 and 2004 were excluded, leaving 10 years for further analysis. The final number of studied patches was 100 (10 patches $\times 10$ years). Mean patch size was 23.89 ha $(\mathrm{SD}=50.38), 48.2 \%$ of which covered $5-$ 10 ha, 30.0\% 10-20 ha, $11.74 \%$ 20-50 ha, 6.09\% 50100 ha and $3.9 \%$ more than 100 ha.

Definition of classes at various thematic resolutions from different spectral indices

In order to measure patch variability from spectral indices, continuous values have to be reclassified into classes, the number of which is dependent on thematic resolution. Three spectral indices were used: NBR (Eq. 1), NDVI (Eq. 2) and TCW (Eq. 3).

$$
\begin{aligned}
\mathrm{NBR}= & \left(\rho_{\mathrm{NIR}}-\rho_{\mathrm{SWIR}}\right) /\left(\rho_{\mathrm{NIR}}+\rho_{\mathrm{SWIR}}\right) \\
\mathrm{NDVI}= & \left(\rho_{\mathrm{NIR}}-\rho_{\mathrm{RED}}\right) /\left(\rho_{\mathrm{NIR}}+\rho_{\mathrm{RED}}\right) \\
\mathrm{TCW}= & 0.15 \mathrm{TM}_{1}+0.18 \mathrm{TM}_{2}+0.33 \mathrm{TM}_{3} \\
& +0.34 \mathrm{TM}_{4}-0.71 \mathrm{TM}_{5}-0.46 \mathrm{TM}_{7}
\end{aligned}
$$

$\rho_{\mathrm{NIR}}, \rho_{\mathrm{RED}}$ and $\rho_{\mathrm{SWIR}}$ are the reflectance of near infrared, red and short-wave infrared bands, respectively. $\mathrm{TM}_{x}$ stands for the channel reflectance of Thematic Mapper Sensor.

Values of each spectral index were grouped into five, nine and 13 classes, thus reflecting three different thematic resolutions. Since each index has

\begin{tabular}{|l|lll|}
\hline Journal : Medium 10980 & Dispatch : 16-1-2010 & Pages : 14 \\
Article No. : 9453 & $\square$ LE & $\square$ TYPESET \\
& MS Code : LAND-09-1926 & $\checkmark \mathrm{CP}$ & $\searrow$ DISK \\
\hline
\end{tabular}


Table 1 Definition of three thematic resolutions (C5, C9 and C13), containing five, nine and 13 classes of spectral values, respectively

\begin{tabular}{llll}
\hline Class & CR5 & C9 & C13 \\
\hline 1 & $0-30$ & $0-15$ & $0-10$ \\
2 & $30-60$ & $15-30$ & $10-20$ \\
3 & $60-90$ & $30-45$ & $20-30$ \\
4 & $90-120$ & $45-60$ & $30-40$ \\
5 & $>120$ & $60-75$ & $40-50$ \\
6 & & $75-90$ & $50-60$ \\
7 & & $90-105$ & $60-70$ \\
8 & & $105-120$ & $70-80$ \\
9 & & $>120$ & $80-90$ \\
10 & & & $90-100$ \\
11 & & & $100-110$ \\
12 & & & $110-120$ \\
13 & & & $>120$ \\
\hline
\end{tabular}

Classes were set up according to ranges of total vegetation cover (values are expressed in percentages). Values were used to calculate patch variability metrics a different range of values which can make the assemblage and interpretation of the classes confusing, linear regression analysis was used to transform spectral data into more understandable and comparable field vegetation cover values (see Table 1). Vegetation was recorded in 21 patches in late summer 2005. We measured total vegetation cover as the sum of all vertical layers above the soil (if more than one layer exists, then vegetation cover can be higher than 100\%). The sampling strategy was based on two factors: time elapsed since the last fire and size of fire scars. Five experimental units $(30 \times 30 \mathrm{~m})$ were randomly distributed within each patch, so that they corresponded spatially to pixels in the satellite images. In patches bigger than 50 ha, up to eight units were defined to account for higher internal heterogeneity. Within each experimental unit, we systematically defined five sampling units of $1 \mathrm{~m}^{2}$ : one in the middle and one in each of the diagonals. Overall, we measured 111 experimental units and 555 sampling units.

Patch variability metrics, general dynamics and scale dependencies

To assess temporal changes in patch variability from the year before the fire event $(x-1)$ until the seventh post-fire year $(x+7)$, we utilized spectral data to calculate three metrics: diversity, richness and evenness, evaluating their role as indicators of environmental variability. Diversity $\left(H^{\prime}\right)$ was measured by means of an adaptation of the Shannon index (Eq. 4; Shannon and Weaver 1949) using spectral class frequency as input data. Richness $(S)$ was calculated as the number of spectral classes in a given patch. Evenness $\left(J^{\prime}\right)$ was the pixel equi-distribution among spectral classes (Eq. 5). The procedure was coded in MatLab (MatLab 2004).

$H^{\prime}=-\Sigma p_{i} \log _{2} p_{i}$

where $p_{i}$ is the probability of each pixel to allocate into each spectral class. $E\{A, B, \ldots, i, \ldots, T\}$ are spectral classes.

$J^{\prime}=H^{\prime} / H_{\max }^{\prime}$

where $H^{\prime}$ is the diversity and $H_{\max }^{\prime}$ is the logarithmic value for richness.

If fire shows a heterogeneous pattern of severity, we expect a high number of spectral classes and, therefore, a high value of diversity, richness and evenness. Parallel reasoning can be applied to vegetation recovery. Conversely, low values of diversity, richness and evenness will be linked to homogeneous patterns originated from very intense/ weak disturbance/recovery processes.

Diversity values $\left(H^{\prime}\right)$ were specifically used to explore the existence of scale-dependencies between spatial patterns and thematic scale. The observation of a phenomenon shows a scale dependency when its mean intensity varies with scale. If a statistical relationship exists between the scale and the variable under analysis, it is possible to undertake scale transfers (i.e. to translate the mean value of the phenomenon to a certain scale from values obtained at another) (Wiens 1989; O'Neill et al. 1991; Auger et al. 1992; Suárez-Seoane and Baudry 2002; Peters et al. 2007). In order to detect scale dependencies, the variance of the annual mean diversity (caused by disturbance or recovery, according to the year: $x-1$, $x, x+1, \ldots, x+7)$ was plotted, for each spectral index, against thematic resolution. A break in the slope variance/scale will indicate differences in the perception of the process across thematic resolutions. We then tested whether the slopes of the lines either sides of the break were significantly different by

\begin{tabular}{|l|lll|}
\hline & Journal : Medium 10980 & Dispatch : 16-1-2010 & Pages : 14 \\
Article No. : 9453 & $\square$ LE & $\square$ TYPESET \\
& MS Code : LAND-09-1926 & $\checkmark \mathrm{CP}$ & $\checkmark$ DISK \\
\hline
\end{tabular}


running ANOVAs. Measures done at the scale where such a rupture is detected are especially relevant because they can be up and down transferred. This analysis allowed the objective choice of a particular thematic resolution to simplify the last part of the study, avoiding redundancy in the results.

\section{Identification of classes of environmental} variability dynamics: relevant drivers

To identify groups of patches exhibiting similar temporal trends in environmental variability, we stored the mean values of Shannon diversity $\left(H^{\prime}\right)$ from the year before the event to the fifth post-fire year for the three spectral indices (NBR, NDVI and TCW) and the thematic resolution selected in the previous step. Here we only used 70 patches, corresponding to fires occurred between 1992 and 2000 (1993 and 1996 were excluded because of low fire occurrence, and the years after 2000 because of a short regeneration period with available data). The implementation of a hierarchical cluster analysis allowed groups of patches with similar dynamics to be defined for each spectral index. These dynamics were clustered using relative Euclidean distance and Ward's method, setting the minimum agglomerative coefficient for group consideration as $80 \%$. Ward's clustering method minimizes within group variance relative to between group variance (van Tongeren 1995). Next, the clusters identified in the previous step were re-grouped into the final classes. Analyses were undertaken in $\mathrm{R}$ statistical package ( $\mathrm{R}$ 2.4.1. 2006).

In order to understand the drivers behind the identified dynamics, we used the Random Forest Algorithm (Breiman 2001) from a set of eleven environmental variables likely to be related to recovery responses (Table 2). These variables describe: (1) frequency of the vegetation types most affected by fire events in the surroundings of a given pixel, (2) topography and (3) patch features related to the fire regime. All these variables were calculated at pixel level and then averaged for each patch (except patch area). Regarding the estimation of fire severity, the standard deviation was also considered because it is related to the spatial variability generated by the disturbance. The Random Forest procedure is based on classification and regression trees. Each node is split by the best predictor selected from a subset of randomly chosen predictors. This method performs very well when compared to other classifiers in discriminant analysis, support vector machines or neural networks and is robust against overfitting (Breiman 2001). In addition, only two parameters must be set by the user (number of variables in the random subset at each node and number of trees in

Table 2 Environmental variables included in the Random Forest analysis

\begin{tabular}{|c|c|}
\hline Variable & Description \\
\hline \multicolumn{2}{|l|}{ Vegetation type } \\
\hline Young_forest & Frequency $(0-1)$ of young forests in a $7 \times 7$ kernel \\
\hline Shrubland & Frequency $(0-1)$ of mixed shrublands in a $7 \times 7$ kernel (dominated by Cytisus scoparius and Genista spp.) \\
\hline Heathland & Frequency $(0-1)$ of heathlands in a $7 \times 7$ kernel (dominated by Erica spp.) \\
\hline \multicolumn{2}{|l|}{ Topography } \\
\hline Elevation & Elevation (m) \\
\hline Slope & Slope (degrees) \\
\hline Solar_rad & Annual solar radiation $\left(\mathrm{MJ} /\left(\mathrm{cm}^{2} \times\right.\right.$ year $\left.)\right)$ \\
\hline Time_rad & Solar insolation duration (hours) \\
\hline Topo_hum & Topographic wetness index (no unit) \\
\hline \multicolumn{2}{|c|}{ Fire-related parameters } \\
\hline Severity_mean & Mean of fire severity (estimated as the change of the NBR value) for pixels included in the patch \\
\hline Severity_std & Standard deviation of fire severity (estimated as the change of the NBR value) for pixels included in the patch \\
\hline Area & Burned patch area (ha) \\
\hline
\end{tabular}

\begin{tabular}{|l|lll|}
\hline Aournal : Medium 10980 & Dispatch : 16-1-2010 & Pages : 14 \\
Article No. : 9453 & $\square$ LE & $\square$ TYPESET \\
MS Code : LAND-09-1926 & $\checkmark$ CP & $\checkmark$ DISK \\
\hline
\end{tabular}


the forest), being the method not very sensitive to their values (Liaw and Wiener 2002). We established the number of trees to be created in 50,000 iterations. The number of variables to be considered in each split that minimized fit error (assessed by the out-ofbag observations) was then calculated by means of an iterative procedure that, in addition, identified and removed outlier observations. Finally, we ran the algorithm and explored the error rate of the resulting model and the importance of each variable measured as: (1) prediction accuracy of the out-of-bag portion of the data compared to other variables (mean decrease in accuracy), and (2) decrease in node impurities (mean decrease Gini).

\section{Results}

Temporal changes in patch variability: the role of spectral indices, patch metrics and thematic scale

Mean diversity $\left(H^{\prime}\right)$ showed similar patterns of temporal change across thematic scale, although values increased when the number of spectral classes was higher (Fig. 3). It must be highlighted that diversity drastically increased within the fire year (associated with the spatial heterogeneity generated

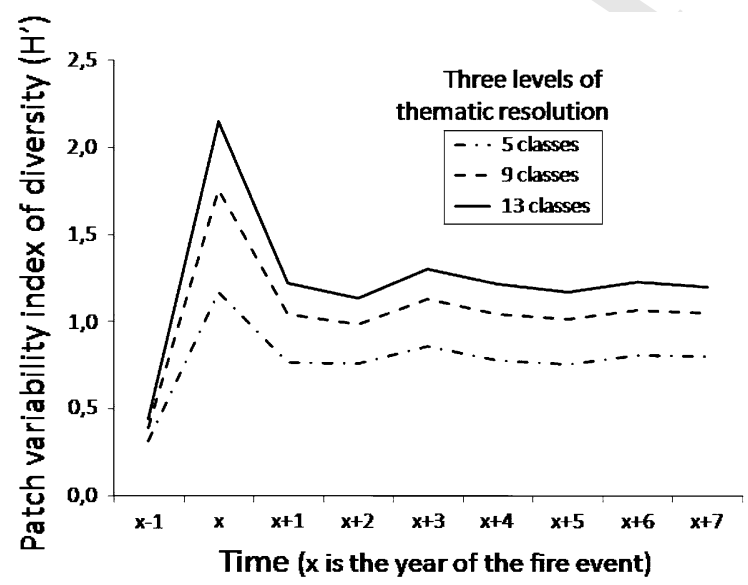

Fig. 3 Temporal changes in patch variability measured as mean diversity $\left(H^{\prime}\right)$ from the year before the event $(x-1)$ to the seventh post-fire year $(x+7)$ at different thematic resolutions. The graph shows the mean values obtained for the three spectral indices (NDVI, NBR and TCW) by disturbance), decreasing then abruptly and stabilizing to the seventh post-fire year. The level of diversity reached at the end of the time span was always higher than that measured in the pre-fire state. Comparing diversity values obtained from the three spectral indices (Fig. 4), we found coherence among them, with NDVI always showing the highest values and TCW the lowest. However, some differences could be observed: NDVI-based values were quite similar for the year of the event and the first post-fire year, slowly decreasing in subsequent years, and almost equalling the values measured the year before the fire. However, TCW and NBR performed dissimilarly, as they showed differences among the year of the event and the following year. Moreover, they revealed higher values of diversity at the end of the recovery time series in comparison with the pre-fire situation. The same results were found for all thematic resolutions.

Analogous conclusions could be drawn for richness $(S)$ and evenness $\left(J^{\prime}\right)$ (Fig. 5) when compared with diversity $\left(H^{\prime}\right)$ (Fig. 4) to characterize changes in patch variability from the three spectral indices. Nevertheless, some slight differences could also be noticed. For the year of the fire event, no differences in evenness were detected by spectral indices, with maximum dissimilarities for richness. Moreover, the year after the fire, only diversity and evenness

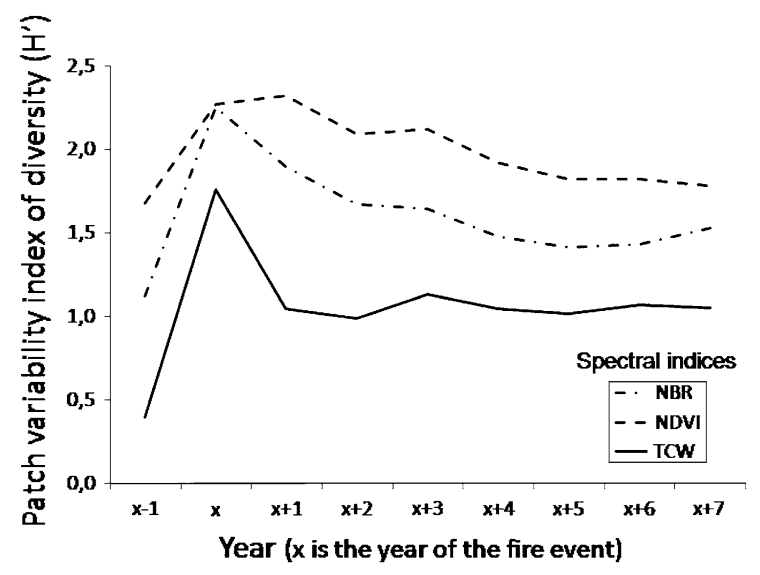

Fig. 4 A comparison of temporal changes in patch variability measured as mean diversity $\left(H^{\prime}\right)$ for the three spectral indices. Years are referenced to the year of the event $(x)$. Values correspond to the nine-class thematic scale as an example

\begin{tabular}{|l|lll|}
\hline & Journal : Medium 10980 & Dispatch : 16-1-2010 & Pages : 14 \\
Article No. : 9453 & $\square$ LE & $\square$ TYPESET \\
MS Code : LAND-09-1926 & $\checkmark \mathrm{CP}$ & $\searrow$ DISK \\
\hline
\end{tabular}




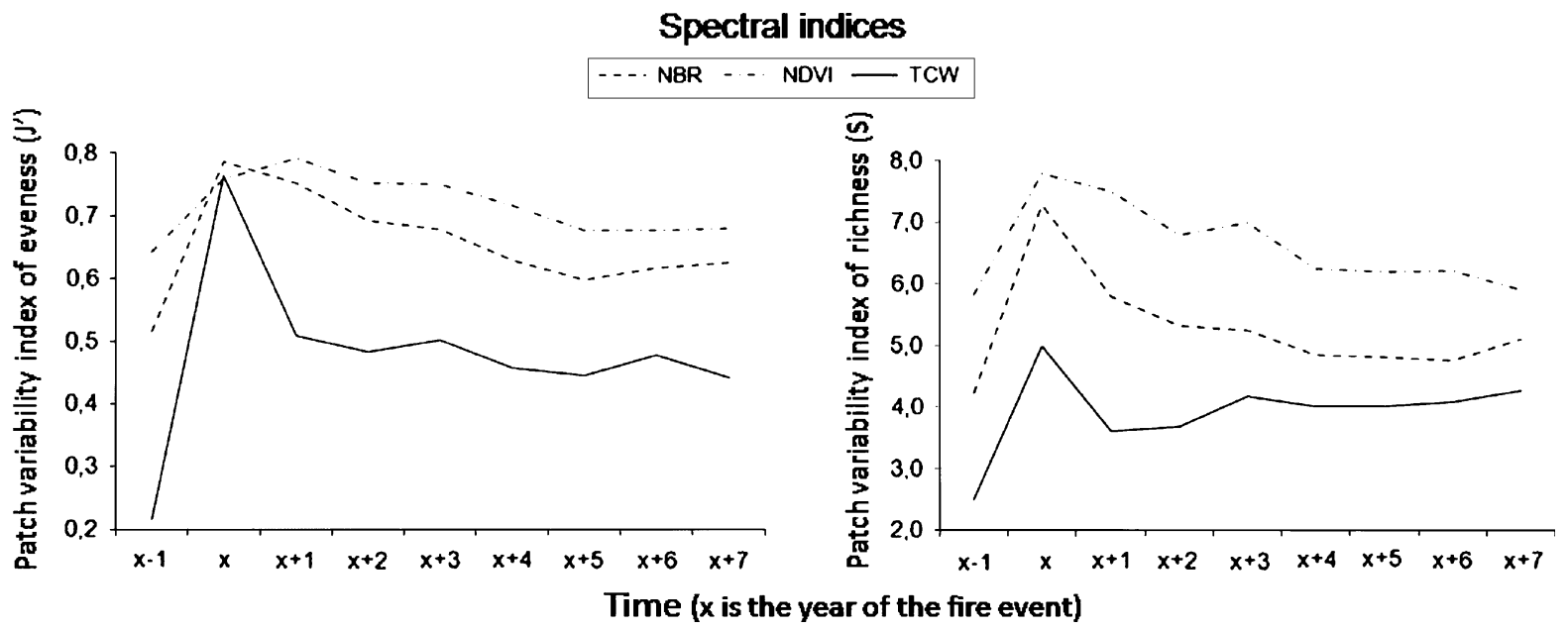

Fig. 5 Temporal changes in patch variability measured as mean evenness $\left(J^{\prime}\right)$ and richness $(S)$ for the three spectral indices. Values correspond to the nine-class thematic scale

measured from NDVI revealed an increase in variability.

Figure 6 plots the variance of the diversity $\left(H^{\prime}\right)$ against thematic resolution, showing different responses for the three spectral indices. TCW did not show any visible pattern in this sense $(F=2.227$, $P=0.155, d f=1)$, while a significant slope break was detected at the 9-classes scale for NDVI $(F=4.679, P=0.046, d f=1)$. NBR also detected a slight but not significantly break at that resolution $(F=3.194, P=0.093, d f=1)$. Therefore, a scale

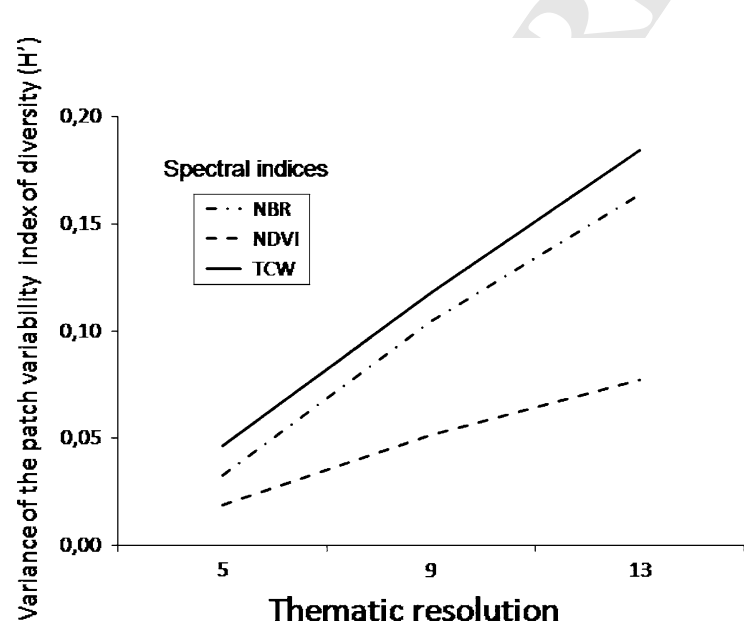

Fig. 6 Variance of the annual mean values of diversity $\left(H^{\prime}\right)$, caused by disturbance or recovery according to the year, plotted against thematic resolution for each spectral index dependency occurred at this intermediate thematic resolution. As a consequence, results obtained at this scale can be transferred to the other proposed thematic levels.

Classes of environmental variability dynamics: relevant drivers

Cluster analysis yielded high agglomerative coefficients, ranging from 0.955 to 0.858 . We obtained nine groups of patches (three groups for each spectral index) with similar dynamics of environmental variability (note that groups based on the TCW index were rejected because of their poor performance). These classes were then grouped into three final classes (Table 3; Fig. 7), which were modelled to predict their occurrence from a set of environmental variables. Patches from classes 1 and 3 showed a strong increase in variability associated with fire events and a subsequent decrease. Class 1 was mainly explained by the altitude (i.e. elevations below 1,300 and above 1,750 m.a.s.l.) and a high spatial heterogeneity of fire severity, while class 3 was associated with low severity fires. Patches from class 2 had the highest variability (before and after fire), reaching a maximum in the first recovery year and showing more consistency through time. They were linked to a high shrubland frequency $(>0.5)$, a low and/or high heathland frequency $(<0.35$ and $>0.75)$ and a high mean fire severity.

\begin{tabular}{|l|lll|}
\hline & Journal : Medium 10980 & Dispatch : 16-1-2010 & Pages : 14 \\
Article No. : 9453 & $\square$ LE & $\square$ TYPESET \\
& MS Code : LAND-09-1926 & $\checkmark \mathrm{CP}$ & $\checkmark$ DISK \\
\hline
\end{tabular}


Table 3 Random Forest models generated for each dynamic identified by the cluster analysis (note that groups based on the TCW index were rejected because of their poor performance)

\begin{tabular}{llllcc}
\hline Index-specific cluster & Final class & $n$ & Total error $(\%)$ & Error absence $(\%)$ & Error occurrence $(\%)$ \\
\hline G1_NBR & Class 1 & 54 & 14.81 & 9.68 & 21.74 \\
G2_NDVI & Class 1 & 52 & 15.38 & 3.22 & 33.33 \\
G2_NBR & Class 2 & 50 & 14.00 & 6.90 & 23.81 \\
G1_NDVI & Class 2 & 53 & 28.30 & 19.30 & 40.90 \\
G3_NBR & Class 3 & 62 & 35.48 & 11.63 & 89.47 \\
G3_NDVI & Class 3 & 59 & 30.51 & 4.76 & 94.1 \\
\hline
\end{tabular}

The final class assigned to each group, the number of observations $(n)$ and the errors are shown for each dynamic

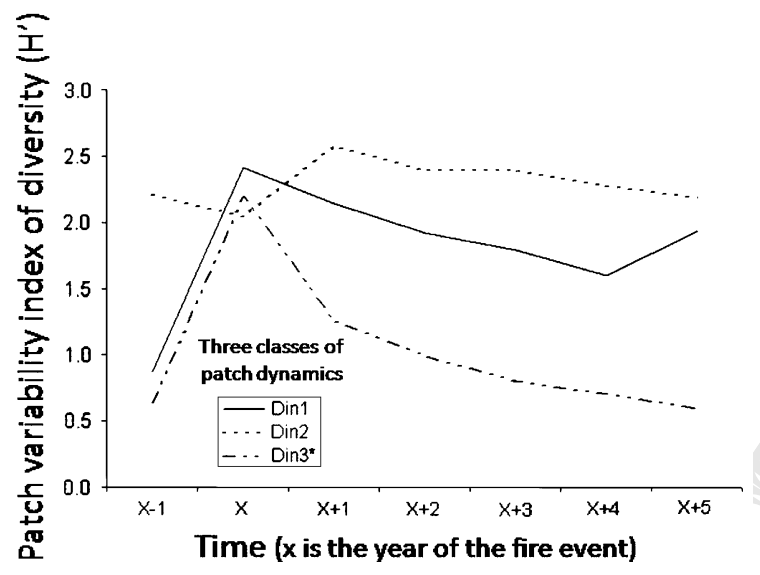

Fig. 7 Dynamic of patch variability for each of the three final classes identified by the cluster analysis

511 Temporal changes in patch variability: the role

512 of the thematic scale and the spectral indices

513 Many authors have studied ecosystem resilience to 514 fire in Mediterranean systems on the basis of 515 estimations of vegetation cover at either local (from 516 field data; e.g. Calvo et al. 2002) or large scales (from 517 raw spectral values derived from remote-sensing 518 sources; e.g. Díaz-Delgado et al. 2002). The present 519 study offers a complementary approach which 520 allowed the characterization of secondary succession 521 in disturbed patches at different thematic scales, on 522 the basis of environmental variability estimated from 523 satellite data. Variability strongly determines the 524 number and distribution of available habitats and, 525 therefore, is a more relevant determinant of species occurrence (Dufour et al. 2006) than vegetation cover in itself. We have shown that, while this system was characterised as highly resilient based on estimates of vegetation cover (Lozano et al., pers. com.), longer time scales are required for these communities to fully recover pre-fire levels of environmental variability. This fact highlights the need to combine different approaches to describe ecological complexity in a more realistic way.

We found that patches regained most of their prefire structural properties during the 2 years following the disturbance. This high resilience to fire has also been found in other Mediterranean areas (Kutiel 1994; Díaz-Delgado et al. 2002; Riaño et al. 2002; Wittenberg et al. 2007). In this sense, Pausas and Vallejo (1999) noted that, within the first year after a fire, vegetation cover reached $52.4 \%$ in north facing slopes. However, in terms of environmental variability, after the initially rapid regeneration, patches do not reach their original levels and slowly continued recovering, with peaks detectable in the third year, which may correlate with an increase in species richness, as noted by other authors (Ne'eman et al. 1995). The level of patch variability achieved at the end of the full time span under study was, in general terms, still higher than that measured in the pre-fire state. This fact suggests that certain structural properties (i.e. diversity) require more time than others (i.e. vegetation cover) to fully recover, probably owing to different rates of ecological processes such as dispersion, colonization or competitive exclusion across the mosaic generated by the disturbance (Hurtt and Pacala 1995; Dufour et al. 2006). According to Keely (1991), most species massively disperse their propagules in spring and summer (prior to autumn

\begin{tabular}{|l|lll|}
\hline & Journal : Medium 10980 & Dispatch : 16-1-2010 & Pages : 14 \\
Article No. : 9453 & $\square$ LE & $\square$ TYPESET \\
MS Code : LAND-09-1926 & $\checkmark \mathrm{CP}$ & $\checkmark$ DISK \\
\hline
\end{tabular}


fires) through passive mechanisms, which may cause a delay in recovery for seeders. Therefore, a primary determinant of similarities between pre- and post-fire states is the dominance of seeders or resprouters in the community, with greater similarities detectable between both states when resprouting species dominate (Keely et al. 2005), as is the case in this study. This is relevant for biodiversity because higher variability implies that more varied environmental conditions and, thus more species, can coexist (Statzner and Moss 2004).

Ecological processes operate at a range of scales in the landscape. Therefore, ecological systems become structured in hierarchies which are specific to each phenomenon, comprising different levels of organization relevant at different scales (Allen and Starr 1982; O’Neill et al. 1986; Urban et al. 1987; Kotliar and Wiens 1990; Wu and David 2002). As a consequence, the scale chosen for examining these processes affects the way in which the system is perceived (He and Gaston 2000). Ecological studies frequently use area-based information derived from field surveys, aerial photography or remote sensing sources. Since the boundaries of these areal units (plots, pixels) are usually arbitrary (or constrained by the resolution of available data), the procedure of defining/changing the scale may show problems related to the Modifiable Areal Unit Problem (Openshaw and Taylor 1981; Jelinski and Wu 1996). In this study, problems may arise from the aggregation of the same set of input data (one for each spectral index) into classes (three thematic resolutions), which often lead to error propagation and controversial results (Wu et al. 2000). This problem should be further explored since it may have significant influences on the determination of relationships among organizational levels and the translation of information across scales. On the other hand, despite its relevance, the analysis of thematic resolution should be done in concert with modifying spatial and, if possible, temporal resolutions. When considering just one scale component, sound conclusions are not warranted. All three scale components are related, making it more relevant to analyze simultaneous scale effects.

In general terms, patch variability (measured as Shannon diversity) due to fire events showed similar patterns of temporal change across thematic resolutions. This is in concordance with Buyantuyev and
Wu (2007), who found that the effects of thematic resolution on many landscape metrics tend to show consistent general patterns. As expected, variability increased with thematic resolution. In this sense, many authors (e.g. Uuemaa et al. 2005) noticed that the effectiveness of metrics to monitor landscape patterns is highly influenced by the way that the map has been defined (i.e. depending on the level of detail of information). Bailey et al. (2007a, b) concluded that data of an intermediate level of thematic resolution are sufficient for general biodiversity monitoring, which is in accordance with our findings. Our patterns of temporal change differed according to the spectral index used to describe the environment, yielding different effects of scale-dependency on landscape perception at different thematic scales. The existence of these scale dependencies can be related to the fact that different species with different life traits perceive environmental variability caused by disturbance/recovery differently (e.g. specialist or poorly mobile species with preferences for homogeneous environments against generalist or highly mobile species living in heterogeneous landscapes; Suárez-Seoane and Baudry 2002).

Trends in environmental variability obtained from the three spectral indices were consistent, allowing for small differences. NDVI appears to perform best and TCW most poorly, a finding contrary to results achieved when evaluating vegetation cover by Lozano et al. (pers. com.). Even if all indices are related to vegetation, each provides different properties. For example, NDVI is more closely linked to photosynthetic activity and NBR to water content and ground signals. Therefore, the former should provide better results when dealing with cover classes (vegetation variability) and the latter when exploring differences between bare soil and vegetation. It seems that photosynthetic activity is homogenised at a lower rate than water content and vegetation cover, which determines the influence of the ground signal.

Diversity, richness and evenness as indicators of environmental variability

Many metrics have been used and "misused" to quantify landscape structure ( $\mathrm{Li}$ and $\mathrm{Wu} 2004$ ) during recent decades. Among them, diversity indices have been demonstrated as particularly appropriate for describing landscapes at detailed thematic resolutions

\begin{tabular}{|l|lll|}
\hline Journal : Medium 10980 & Dispatch : 16-1-2010 & Pages : 14 \\
Article No. : 9453 & $\square$ LE & $\square$ TYPESET \\
MS Code : LAND-09-1926 & $\checkmark$ CP & $\checkmark$ DISK \\
\hline
\end{tabular}


while others, such as grain and dominance, work better at low thematic resolutions (Bailey et al. 2007a, b). Here, we explored three diversity-based metrics (i.e. Shannon diversity, richness and evenness), founding similar trends of change through the study period. This contrasts with Yue et al. (2005) who found different tendencies when different diversity metrics were used to measure landscape patterns. In our case, we can conclude that the three metrics are redundant if used in combination, as they are providing similar information. This makes them useful and coherent indicators ( $\mathrm{Li}$ and $\mathrm{Wu} 2004$ ) of environmental variability only if used independently of each other, with richness providing the least timeconsuming measure. However, some differences between the three patch metrics were detected according to the behaviour of the three spectral indices when measuring the variability induced immediately after the disturbance event, with greater similarity between richness and diversity than evenness. Such specific differences between indices can be due to the water content of the vegetation, which is related to TCW values, and which seems to be more spatially homogeneous before and after the fire event than the features related to NDVI (photosynthesis) and NBR (ground signal, water content and others) (Lozano et al. 2007b, 2008).

Classes of environmental variability dynamics: relevant drivers

A closer investigation of patch variability dynamics has shown three main classes of change which were strongly related, among other factors, to the severity of fire event, which seriously affects the resprouting capabilities of shrubland (Keely et al. 2005) and, therefore, determines post-fire recovery. Class 1 of patch variability dynamic was associated with the most heterogeneous pattern of fire severity, which was responsible for the creation of a mosaic of patches affected to differing degrees, as reflected in the highest peak in environmental variability during the fire event. The other relevant driver explaining this dynamic was elevation, probably because it is closely linked with radiation and precipitation, both largely determining vegetation recovery (DíazDelgado et al. 2002). Radiation is less favourable to vegetation development because is associated with large water losses through evapotranspiration (an effect not compensated for by higher photosynthetic activity) (Röder et al. 2008), while precipitation is obviously related to higher water availability and, therefore, more rapid post-fire recovery. Class 2 occurred in patches where intense fires homogenized the heterogeneous pre-fire spatial pattern, which very quickly recovered (after 1 year). This may be explained by: (1) the positive relationship between fire severity and shrubs found by Keely et al. (2005), who theorized that this may be due to the effect of high temperatures on the stimulation of dormant seed banks, which benefits non-resprouting species; (2) scarce vegetation cover before the fire or presence of rocky formations in the mosaic, (3) initially heterogeneous water availability (e.g. patches close to a water course) or (4) human-related changes. Class 3 was characterised by very rapid recovery associated with low severity fires; in this case, temporary habitats created by the fire do not persist for long and are rapidly recolonized.

\section{General conclusions}

Monitoring spatial and temporal changes in patch variability on a regular basis is a valuable tool for defining conservation measures for species living in fire-affected areas. This paper provides a methodological framework useful for environmental managers to design policies related to vegetation post-fire recovery management on the basis of remote-sensed data. The method, which explicitly considers spatial heterogeneity, allowed us to (1) identify general trends in environmental variability; (2) understand the most crucial environmental factors affecting those trends; (3) identify levels of organisation within the system and scale dependencies where thematic scale transfers are possible. The combination of ecological (i.e. patch variability metrics) and spectral indices has shown promising results that should be further explored.

Acknowledgments This work was supported by the Spanish Ministry of Education and Science under the research project REN2002-04463-C02-01 and research grant BES-2003-3130 awarded to F. J. Lozano. The authors would like to thank L. Calvo and J. M. Álvarez (Área de Ecología, University of León), D. Lozano (Telefónica I+D), and J. L. Gutierrez (Lago de Sanabria and Surroundings Natural Park, staff) for their valuable comments that increased the quality of the manuscript. Finally

$\begin{array}{lll}\text { Journal : Medium 10980 } & \text { Dispatch : 16-1-2010 } & \text { Pages : } 14 \\ \text { Article No. : } \mathbf{9 4 5 3} & \square \text { LE } & \square \text { TYPESET } \\ \text { MS Code : LAND-09-1926 } & \checkmark \text { CP } & \checkmark \text { DISK }\end{array}$


we wish to thank the Fire Dept. of the Environmental Section, Junta de Castilla y León, for their support to this study by providing digital geographical data.

\section{References}

Allen TFH, Starr TB (1982) Hierarchy: perspectives for ecological diversity. University of Chicago Press, Chicago

Arnan X, Rodrigo A, Retana J (2006) Post-fire recovery of Mediterranean ground ant communities follows vegetation and dryness gradients. J Biogeogr 33:1246-1258

Arnot C, Fisher PF, Wadsworth R, Wellens J (2004) Landscape metrics with ecotones: pattern under uncertainty. Landscape Ecol 19:181-195

Auger P, Baudry J, Fournier F (eds) (1992) Hiérarchies et échelles en écologie. Naturalia Publications, Cahors

Bailey D, Herzog F, Augenstein I, Aviron S, Billeter R, Szerencsits E, Baudry J (2007a) Thematic resolution matters: Indicators of landscape pattern for European agro-ecosystems. Ecol Indic 7:692-709

Bailey D, Billeter R, Aviron S, Schweiger O, Herzog F (2007b) The influence of thematic resolution on metric selection for biodiversity monitoring in agricultural landscapes. Landscape Ecol 22:461-473

Baudry J, Burel F (1997) Agricultural landscape dynamics: implications for species movements. In: Cooper A, Power J (eds) Species dispersal and land use processes. Proceedings of the sixth annual IALE (UK) conference. University of Ulster, Coleraine, pp 3-10

Bond WJ, van Wilgen B (1996) Fire and plants. Chapman and Sons, New York

Breiman L (2001) Random forests. Mach Learn 45:5-32

Buyantuyev A, Wu J (2007) Effects of thematic resolution on landscape pattern analysis. Landscape Ecol 22:7-13

Calvo L, Tárrega R, De Luis E (2002) Secondary succession after perturbations in a shrubland community. Acta Oecologica 23:393-404

Chávez PS Jr (1996) Image-based atmospheric correctionsrevisited and improved. Photogramm Eng Remote Sens 62:1025-1036

Connell JH (1978) Diversity in tropical rain forests and coral reefs. Science 199:1302-1310

Crist EP, Cicone RC (1984) A physically-based transformation of thematic mapper data-the TM Tasseled Cap. IEEE Trans Geosci Remote Sens 22:256-263

De Luis M, Raventós J, Wiegand T, González-Hidalgo JC (2008) Temporal and spatial differentiation in seedling emergence may promote species coexistence in Mediterranean fire-prone ecosystems. Ecography 31:620-629

Díaz-Delgado R, Pons X (2001) Spatial patterns of forest fires in Catalonia (NE Spain) along the period 1975-1995: analysis of vegetation recovery after fire. For Ecol Manage 147:67-74

Díaz-Delgado R, Lloret F, Pons X, Terradas J (2002) Satellite evidence of decreasing resilience in Mediterranean plant communities after recurrent wildfires. Ecology 83:22932303

Dufour A, Gadallah F, Wagner HH, Guisan A, Buttler A (2006) Plant species richness and environmental heterogeneity in a mountain landscape: effects of variability and spatial configuration. Ecography 29:573-584

Dumbrell AJ, Clark EJ, Frost GA, Randell TE (2008) Changes in species diversity following habitat disturbance are dependent on spatial scale: theoretical and empirical evidence. J Appl Ecol 45:1531-1539

Gilabert MA, Conese C, Maselli F (1994) An atmospheric correction method for the automatic retrieval of surface reflectances from TM images. Int $\mathrm{J}$ Remote Sens 15:2065-2086

Groeneveld J, Enright NJ, Lamont BB (2008) Simulating the effects of different spatio-temporal fire regimes on plant metapopulation persistence in a Mediterranean-type region. J Appl Ecol 45:1477-1485

Hall FG, Strebel DE, Nickeson JE, Goetz SJ (1991) Radiometric rectification: toward a common radiometric response among multidate, multitsensor images. Remote Sens Environ 35:11-27

Hammer KC, Hill JK (2000) Scale-dependence effects of habitat disturbance on species richness in tropical forests. Conserv Biol 14:433-444

He F, Gaston KJ (2000) Occupancy-abundance relationships and sampling scales. Ecography 23:503-511

Hurtt GC, Pacala SW (1995) The consequences of recruitment limitation: reconciling chance, history and competitive differences between plants. J Theor Biol 176:1-12

Huston MA (1994) Biological diversity: the coexistence of species on changing landscapes. Cambridge University Press, Cambridge

Jelinski DE, Wu J (1996) The modifiable areal unit problem and implications for landscape ecology. Landscape Ecol 11:129-140

Keely JE (1991) Seed germination and life history syndromes in the California chaparral. Bot Rev 57:81-116

Keely JE, Fotheringham CJ, Baer-Keely M (2005) Determinants of post-fire recovery and succession in Mediterranean-climate shrublands of California. Ecol Appl 15:1515-1534

Key CH, Benson NC (1999) Measuring and remote sensing of burn severity. In: Neuenschwander LF, Ryan KC (eds) Proceedings of the joint fire science conference and workshop, vol II. University of Idaho and International Association of Wildland Fire, Moscow

Kotliar NB, Wiens JA (1990) Multiple scales of patchiness and patch structure: a hierarchical framework for the study of heterogeneity. Oikos 59:253-260

Kutiel P (1994) Fire and ecosystem heterogeneity: a Mediterranean case study. Earth Surf Process Landforms 19:187194

Lavorel S (1999) Ecological diversity and resilience of Mediterranean vegetation to disturbance. Divers Distrib 5:3-13

Levin SA (1992) The problem of pattern and scale in ecology. Ecology 73:1943-1967

Li H, Wu J (2004) Use and misuse of landscape indices. Landscape Ecol 19:389-399

Liaw A, Wiener M (2002) Classification and regression by Random Forest. R News 2/3:1609-3631

Lozano FJ, Suárez-Seoane S, De Luis E (2005) Vegetation dynamics after fire: opportunities of the combined use of fire detection and ecological indices: a case study in West Spain. In: Riva $\mathrm{J}$ et al (eds) Proceedings of the 5th

\begin{tabular}{|l|lll|}
\hline & Journal : Medium 10980 & Dispatch : 16-1-2010 & Pages : 14 \\
Article No. : 9453 & $\square$ LE & $\square$ TYPESET \\
& MS Code : LAND-09-1926 & $\checkmark \mathrm{CP}$ & $\checkmark$ DISK \\
\hline
\end{tabular}


international workshop on remote sensing and GIS applications to forest fire management: fire effects assessment. Universidad de Zaragoza, Spain, pp 269-273

Lozano FJ, Suárez-Seoane S, De Luis E (2007a) Estudio comparativo de los regímenes de fuego en tres espacios naturales protegidos del oeste peninsular mediante imágenes Landsat. Rev Esp Teledetec 26:77-86

Lozano FJ, Suárez-Seoane S, De Luis E (2007b) Assessment of several spectral indices derived from multi-temporal Landsat data for fire occurence modelling. Remote Sens Environ 107:533-544

Lozano FJ, Suárez-Seoane S, De Luis E (2008) A multi-scale approach for modelling fire occurence probability using satellite data and classification trees. A study case in a mountainous Mediterranean region. Remote Sens Environ 112:708-719

Mackey RL, Currie DJ (2001) The diversity-disturbance relationship: is it generally strong and peaked? Ecology 82:3479-3492

Magurran AE (2004) Measuring biological diversity. Blackwell Science Ltd, Oxford

Markham BL, Barker JL (1986) Landsat MSS and TM postcalibration dynamic ranges, exatmospheric reflectances and at-satellite temperatures. EOSAT Landsat Technical Notes 1:3-8

MatLab (2004) MatLab Version 7. The Language of technical computing. Release 14. The MathWorks, Natick

Moran MS, Jackson RD, Slater PN, Teillet PM (1992) Evaluation of simplified procedures for retrieval of land surface reflectance factors from satellite sensor output. Remote Sens Environ 41:169-184

Moreno JM, Vázquez A, Vélez R (1998) Recent history of forest fires in Spain. In: Moreno JM (ed) Large forest fires. Backhuys Publishers, Netherlands, pp 159-185

Ne'eman G, Lahav H, Izhaki I (1995) Recovery of vegetation in a natural east Mediterranean pine forest on Mount Carmel, Israel as affected by management strategies. For Ecol Manage 75:15-26

O'Neill RV, DeAngelis DL, Waide JB, Allen TFH (1986) A hierarchical concept of ecosystems. Princeton University Press, Englewood Cliffs

O'Neill RV, Turner SJ, Cullinan VI, Coffin DP, Cook T, Conley W, Brunt J, Thomas JM, Conley MR, Gosz J (1991) Multiple landscape scales: an intersite comparison. Landscape Ecol 5:137-144

Openshaw S, Taylor PJ (1981) The modifiable areal unit problem. In: Wrigley N, Bennett R (eds) Quantitative geography: a British view. Routledge and Kegan Paul Ed, London, pp 60-69

Palá V, Pons X (1995) Incorporation of relief into geometric correction based on polynomials. Photogramm Eng Remote Sens 7:935-944

Pausas JG, Vallejo R (1999) The role of fire in European Mediterranean ecosystems. In: Chuvieco E (ed) Remote sensing of large wildfires. Springer, Berlin, pp 2-16

Peet RK (1974) The measurement of species diversity. Ann Rev Ecol System 5:285-307

Peters DPC, Bestelmeyer BT, Turner MG (2007) Cross-scale interactions and changing pattern-process relationships: consequences for system dynamics. Ecosystems 10:790796
$\mathrm{R}$ 2.4.1. (2006) The $\mathrm{R}$ foundation for statistical computing

Riaño D, Chuvieco E, Ustin S, Zomer R, Dennison P, Roberts D, Salas J (2002) Assessment of vegetation regeneration after fire through multitemporal analysis of AVIRIS images in the Santa Monica Mountains. Remote Sens Environ 79:60-71

Riaño D, Chuvieco E, Salas J, Aguado I (2003) Assessment of different topographic corrections in Landsat-TM data for mapping vegetation types. IEEE Trans Geosci Remote Sens 41:1056-1061

Röder A, Hill J, Duguy B, Alloza JA, Vallejo R (2008) Using long time series of Landsat data to monitor fire events and post-fire dynamics and identify driving factors. A case study in the Ayora region (eastern Spain). Remote Sens Environ 112:259-273

Rouse JW, Haas RH, Schell JA, Deering DW (1973) Monitoring vegetation systems in the great plains with ERTS. Proceedings of the third ERTS Symposium 1, NASA SP-351, pp 309-317

Saura S (2004) Effects of remote sensor spatial resolution and data aggregation on selected fragmentation indices. Landscape Ecol 19:197-209

Schröter D et al (2005) Ecosystem service supply and vulnerability to global change in Europe. Science 310:13331337

Shannon CE, Weaver W (1949) The mathematical theory of communication. University of Illinois Press, Urbana

Shao G, Wu J (2008) On the accuracy of landscape pattern analysis using remote sensing data. Landscape Ecol 23:505-511

Statzner B, Moss B (2004) Linking ecological function, biodiversity and habitat: a mini-review focusing on older ecological literature. Basic Appl Ecol 5:97-106

Suárez-Seoane S, Baudry J (2002) Scale dependence of spatial patterns and cartography on the detection of landscape change: relationships with species' perception. Ecography 25:499-511

Suárez-Seoane S, Osborne PE, Baudry J (2002) Responses of birds of different biogeographic origins and habitat requirements to agricultural land abandonment in northern Spain. Biol Conserv 105:333-344

Teillet PM, Guindon B, Goodeonugh DG (1982) On the slopeaspect correction of multispectral scanner data. Can J Remote Sens 8:84-106

Trabaud L, Galtié JF (1996) Effects of fire frequency on plant communities and landscape pattern in the Massif des Aspres (S. France). Landscape Ecol 11:215-224

Urban DL, O'Neill RV, Shugart HH (1987) Landscape ecology. A hierarchical perspective can help scientists understand spatial patterns. Bioscience 37:119-127

Uuemaa E, Roosaare J, Mander U (2005) Scale dependence of landscape metrics and their indicatory value for nutrient and organic matter losses from catchments. Ecol Indic 5:350-369

van Tongeren OFR (1995) Cluster analysis. In: Jongman R et al (eds) Data analysis in community and landscape ecology. Cambridge University Press, Cambridge, pp 174-212

Wiens JA (1989) Spatial scaling in ecology. Func Ecol 3:385-397 Wittenberg L, Malkinson D, Beeri O, Halutzy A, Tesler N (2007) Spatial and temporal patterns of vegetation

\begin{tabular}{|l|lll|}
\hline & Journal : Medium 10980 & Dispatch : 16-1-2010 & Pages : 14 \\
Article No. : 9453 & $\square$ LE & $\square$ TYPESET \\
MS Code : LAND-09-1926 & $\checkmark \mathrm{CP}$ & $\checkmark$ DISK \\
\hline
\end{tabular}


recovery following sequences of forest fires in a Mediterranean landscape, Mt. Carmel Israel. Catena 71:76-83

Wu J (2004) Effects of changing scale on landscape pattern analysis: scaling relations. Landscape Ecol 19:125-138

Wu J, David JL (2002) A spatially explicit hierarchical approach to modeling complex ecological systems: theory and applications. Ecol Model 153:7-26

Wu J, Jelinski DE, Luck M, Tueller PT (2000) Multiscale analysis of landscape heterogeneity: scale variance and pattern metrics. Geogr Inf Sci 6:6-19
Wu J, Shen W, Sun W, Tueller PT (2002) Empirical patterns of the effects of changing scale on landscape metrics. Landscape Ecol 17:761-782

Yue TX, Liu JY, Li ZQ, Chen SQ, Ma SN, Tian YZ, Ge F (2005) Considerable effects of diversity indices and spatial scales on conclusions relating to ecological diversity. Ecol Model 188:418-431 